\title{
Effects of neuro-linguistic programming course on job stress, positive organizational behavior and job motivation in physical education teachers
}

\author{
Mohammad Reza Anjomshoa ${ }^{1 \mathrm{ABCDE}}$, Hasan Fahim Devin ${ }^{1 \mathrm{ABCD}}$, Mohammad Reza Esmailzadeh ${ }^{1 \mathrm{ACD}}$, \\ Mohammad Keshtidar ${ }^{2 A C D}$ \\ ${ }^{l}$ Department of Physical Education and Sport Sciences, Mashhad Branch, Islamic Azad University, Mashhad, Iran \\ ${ }^{2}$ Faculty of Sport Sciences, Ferdowsi University of Mashhad, Mashhad, Iran
}

Authors' contributions: A - Study design; B - Data collection; C - Statistical analysis; D - Manuscript Preparation; E - Funds Collection.

\begin{abstract}
Purpose:

The purpose of this study was to investigate the effects of a neuro-linguistic programming course on job stress, positive organizational behavior and job motivation among physical education teachers of Khorasan Razavi province of Iran.

Material: $\quad$ The statistical population of the study consisted 150 physical education teachers of Khorasan Razavi province. 40 physical education teachers were selected as statistical sample. At first, the questionnaires of job stress of Steinmetz (2002), Luthans Psychological Capital questionnaire (2007) and Hackman job motivation questionnaire (1976) were administered to the subjects and then the subjects received the presented protocol. At the end of the course questionnaires were re-presented. Data were analyzed using repeated measures ANOVA and Bonferroni post hoc test at $5 \%$ error level.

Results: $\quad$ The results showed that neuro-linguistic programming had a significant increase in job motivation and positive organizational behavior and a significant decrease in teachers' job stress $(P<0.001)$. There was no significant change in job motivation, positive organizational behavior and job stress in the control group $(P<0.05)$.

Conclusions: $\quad$ According to the findings, it can be concluded that to increase the job motivation, these course can be used and affect the motivation and job stress of the employees.

Keywords: $\quad$ neuro-linguistic programming, job stress, positive organizational behavior, job motivation.
\end{abstract}

\section{Introduction}

The most important part of organizations is their human resources. First of all, human resources should be examined and their motivations and potentials should be recognized and a reasonable degree of their satisfaction or success should be provided, because without understanding the motivations and desires of the human resources, they cannot be forced to serve and work beneficially. Regarding the necessity of understanding the motivations of human resources, it is also important that almost all human beings spend most of their lives in organizational environments, and work is an experience that most of the people gain, since our society is strongly dependent on the effective and efficient performance of its organizations. It is, therefore, necessary to have an evoked force. Today, the importance of motivation in performance is obvious and widely known, and it is an issue of concern in human resource management [1-3]. Therefore, any effort for better managing human beings requires discovering basic needs, understanding the behavioral motivations of individuals, and reactions of different group works. Therefore, identifying one in an organization and understanding the basics of his/ her behavior, and consequently, motivating him/her is in fact one of the most vital and most difficult tasks of managers [2]. If we want people to do their jobs willingly and eagerly in the workplace, we need to be aware of

\footnotetext{
(c) Anjomshoa M.R., Fahim Devin Y., Esmailzadeh M.R.

Keshtidar M., 2020

doi:10.15561/26649837.2020.0302
}

these needs and desires, and create an environment in which these requests and desires are fulfilled, and the motivations are satisfied. In such an environment that the emotional, psychological and social needs of employees are taken into enough consideration, it is expected that individuals will feel involved and attached to their work environment, their interest and motivation for working will be increased, and thus, they will work with more morale and satisfaction $[4,5]$.

Job stress is one of the factors that is a problem for many organizations and their employees. Not so long ago, nervousness was considered to be a human disease, whereas today, behavioral science experts believe that nervousness is not a disease, but an inevitable fact that a degree of which is useful for pursuing organizational goals and also personal and group objectives and people's efforts should be directed at controlling, directing, and properly using such pressures, not at resisting them $[4,6]$. Experts define job stress and factors causing job stress differently. Lutanz has considered stress as a response a person gives as a psychological or physical behavior in order to adapt to an external situation different from the normal one. In this definition, stress is the reaction of an individual to the threatening situations in the environment. Hans defines stress as a reaction to external unfavorable or unexpected factors, or simply, to the disturbance in the human body's adaptation system to the external environment. From his point of view, whenever the balance and adaptation of the organism are eliminated due to disruptive factors, the 
individual becomes stressed out [7].

On the other hand, there is a fundamental change in the attitude of some psychologists. The new focus and direction of this attitude is called perfectionism or health psychology, which deals with the healthy aspect of human nature, not the unhealthy aspect of it. This new approach, also called positive psychology, has recently attracted the attention of many researchers in the field of organization and management, thus creating a new movement called Positive Organizational Behavior. Like Positive Psychology, Positive Organizational Behavior does not claim to have achieved a new breakthrough in Positive Behavior, but rather points to the need to more focus on theorizing, researching, and effectively applying employees' positive states, traits, and behaviors [8]. After physical capital, human resources consisting of the knowledge, skills, and experiences of employees, and ultimately social capital, was raised as a set of networks of individual relationships with others [8].

Considering these, research needs to be done to enhance positive organizational motivation and reduce management job stress in organizations. Therefore, it is necessary to use appropriate methods for this issue. In this regard, verbal neural planning has been the subject of a lot of research studies in the past few decades. The impact of this concept on communication skills and job skills has been frequently investigated by researchers and its role in managing and improving communication has been questioned by many researchers. Neuro-linguistic programming (NLP) is a revolutionary approach to the development of human and individual communication. Some call it the science and art of individual success or mental experiences. This approach offers the highest level of skills in communicating between individuals and practical ways to transform thinking and behavior $[8,9]$. Through NLP, individual and organizational goals can be achieved faster and more effectively than ever before. Using NLP techniques, mental resources will be employed, and the use of the word element will facilitate the achievement of goals, and mental status will be achieved and beliefs and values will be aligned to achieve the desired results. Neuro-linguistic programming is the study of the structure of mental and logical experience $[10,11]$. Neuro-linguistic programming raises selfawareness and provides new insights into the relationship between body and mind. In this regard, the awareness of the system of representations, goals, beliefs, and values of the individual has been developed and leads to the awareness of others, and based on these levels of awareness, the individual's communicative action becomes more effective and the individual support and communication networks are developed, and beliefs of self-efficacy and self-esteem are changed [12].

In this regard, Troesch and Bauer [6] believe that self-efficacy has a greater impact on job stress, and Howard [8] associates positive self-assessment with positive psychological capital. Also, Jancenelle et al. [13] believe that in the economic environment, psychological capital and market approach have a major impact on the economic conditions and performance of lending companies and the behavior of borrowers. Ray et al. [14] correlated job stress and quality of work life, and $\mathrm{Wu}$ and Park [15] has found a significant impact of the satisfaction with expertise of nursing students on psychological capital and professional values, and Pan et al. [8] has found the impact of psychological capital and positive organizational behavior on job attachment and interest in the workplace meaningful. Younesian [16] found that college students' psychological capital is related with learning empowerment and interaction. Ahmadi et al. [7] emphasized on the effect of neuro-verbal programming training on depression, and Kajbaf et al. [17] confirmed the effect of this training on marital adaptability, and Mikaili and colleagues [18] verified its effect on mental health of nurses. Ashok \& Santakumar [19] found verbal neural planning to improve learning effective, and Thompson et al. [20] found it effective in improving individual and organizational performance, and Grosu et al. [21] found it effective in decision. Lashkarian and Sayyadian [22] also found the effect of verbal neural planning to increase motivation and Florina et al. [23] found it effective in reducing anxiety. Yemm [24] believes that NLP can help in the business world. However, there are ways that NLP can easily harm companies.

Job motivation in physical education teachers as a key stimulator of teachers' performance is the missing link in developing physical education in schools. Physical education teachers deal with a variety of problems inside and outside the organization that increase job stress and decrease job motivation in teachers. Therefore, in addition to trying to solve the livelihoods and organizational problems of physical education teachers, appropriate measures should be taken to improve their job motivation to perform better. Therefore, it seems necessary to examine strategies for promoting motivation and positive organizational behaviors and reduce job stress in teachers. Background research shows that so far research has been done on verbal neural planning and organizational behavior and job motivation variables, and the positive effects of these variables have been reported. Also, developing verbal neural planning and managing emotions in controlling individuals' internal emotions can improve their feelings about themselves and the organization and create a positive sense of belonging that enhances employees' job satisfaction and motivation, which is the missing link of the organization. The performance of physical education teachers in training education plays an important role in the development of sports in the country and can provide the basis for further development of sports in the country. Sport science is directly linked to the development of students' physical and mental health and the training of healthy lifestyles. Assessing primary physical health and improving students' health indicators and, to a higher level, improving their motor and athletic skills are among the duties of a physical education teacher. Meanwhile, a knowledgeable physical education teacher plays an important role in identifying the country's future champions, with his or her strategies of recognizing 
talented students. However, in order to develop the job motivation of the teachers, appropriate measures must be taken inside and outside the organization. Therefore, it is necessary to study the factors affecting their job motivation and conduct research in this area. Thus, the purpose of this study was to determine if verbal neuroscience training is effective on positive organizational behavior and job stress and how does this effect on job motivation?

\section{Material and methods}

\section{Participants}

The statistical population of the study consisted 150 physical education teachers of Khorasan Razavi province. 40 physical education teachers with the highest scores in job stress were selected as statistical sample.

\section{Research Design}

The present study is a quasi-experimental research with pre-test and post-test in control and experimental groups. In terms of purpose, this research is a kind of applied research.

Statistical sample (40 teachers) randomly divided in two control and experimental groups. Then experimental group received neuro-linguistic programming based on the research goals for ten sessions. The teachers in the control group did not receive any intervention. The training protocol is as follows (tabl. 1).

At the end of the course, job stress questionnaires of Steinmetz [25], and psychological capital questionnaire of Luthans [26] and job motivation questionnaire of Hackman [27] were re-presented to the subjects, and their indices were assessed.

The validity of the research questionnaires has been evaluated in various research studies and they were found to be standard tools having validity. The Job Stress Questionnaire of Steinmetz [25], consisted of 36 questions and was designed on a Likert scale. A score of 150 to 180 indicates a high level of job stress, a score of 110 to 149 indicates a moderate level of job stress, a score of 80 to 109 indicates a low level of job stress, and a score below 79 indicates a very low level of job stress. The validity of the questionnaire was confirmed in several researches and its reliability was calculated as 0.89 by Cronbach's alpha test. The Luthans [26], psychological capital questionnaire was designed on a Likert scale and consisted of 20 questions. A score of 80-100 indicates a high level, 60-79 indicates a good level, 40-59 indicates a moderate level, and a score below 39 indicates a low level of psychological capital (positive organizational behavior). This tool is standard and its validity has been confirmed by various studies and its reliability was calculated by Cronbach's alpha of 0.91. Hackman [27], Job Motivation Questionnaire contains 25 questions on the Likert scale, with a score of 100 to 125 indicating a very good level of job motivation, a score of 75 to 99 indicating a good level of job motivation, a score of 55 to 74 indicating a low level of job motivation, and a score of less than 54 indicating a very low level of job motivation. The validity of this questionnaire has been confirmed by various research studies, and it is a standard tool and its reliability was calculated and confirmed by Cronbach's alpha of 0.90 .

\section{Statistical Analysis}

The analysis was performed at both descriptive and inferential levels. In descriptive level, mean and standard deviation indices, and in the inferential level, variance with repeated measures of $(2 \times 2)$ were used for analysis. Tests were performed at 5\% error level using SPSS software version 25 . The assumptions of variance analysis model were examined with repeated measures such as the normality of error distribution by Shapiro-Wilk Test, the homogeneity of variance error by Levin test, and the homogeneity of covariance by Box test on the data of the present study.

\section{Results}

The normality of the error distribution was evaluated by Shapiro-Wilk Test, and based on the results of this test, the variable of job stress was $(p=0.794)$ in pretest, and it was $(\mathrm{p}=0.554)$ in post-test, the variable of job motivation was $(P=0.485)$ and it was $(p=0.696)$ in post-test, and the variable of positive organizational behavior was $(\mathrm{p}=0.134)$ in pre-test, and $(\mathrm{p}=0.893)$ in post-test, and no evidence was found to reject the assumption of normality. The homogeneity of variance error between groups was also evaluated by Levin test. Based on the results of this test, the assumption of homogeneity of variance between groups for the variable of job stress was $(p=0.065)$ in pre-test, and $(p=0.05)$ in post-test, for the variable of job motivation, it was (p$0.416)$ in pre-test, and $(\mathrm{p}=0.416)$ in post-test, and for

Table 1. Training Protocol

\begin{tabular}{ll} 
Sessions & Learning content \\
\hline First and Second Sessions & Introductions and Introductory Concepts of Neuro-linguistic programming \\
Third Session & Teaching how to set and reach goals \\
Fourth Session & Teaching to create a sense of self-esteem and self-love \\
Fifth Session & Concepts of control and flexibility in problems and mind control to solve crises \\
Sixth Session & Concepts of closed mindedness and readiness to accept new beliefs \\
Seventh Session & Giving yourself motivation and hope and optimism \\
Eighth Session & Concepts of self-efficacy and utilization of your inner abilities \\
Ninth Session & Teaching strategies to improve self-confidence \\
Tenth Session & Getting familiar with how to make effective relationship and the ways of reaching it
\end{tabular}


Table 2. The mean and standard deviation of the scores of job stress, job motivation and positive organizational behavior in two groups in pre-test and post-test and repeated measures of ANOVA test results.

\begin{tabular}{|c|c|c|c|c|c|c|c|c|}
\hline \multirow[b]{2}{*}{ Variable } & \multirow[b]{2}{*}{ Group } & \multicolumn{2}{|l|}{ Pre-test } & \multicolumn{2}{|c|}{ Post-test } & \multicolumn{3}{|l|}{ P Value } \\
\hline & & Mean & $\begin{array}{l}\text { SD } \\
\text { deviation }\end{array}$ & Mean & $\begin{array}{l}\text { SD } \\
\text { deviation }\end{array}$ & Group & track & $\begin{array}{l}\text { Interaction } \\
\text { Effect }\end{array}$ \\
\hline \multirow{2}{*}{ Job Tension } & Experimental & 147.75 & 8.97 & 74.60 & 6.68 & $<0.001$ & $<0.001$ & $<0.001$ \\
\hline & Control & 145.80 & 5.72 & 146.45 & 5.40 & $(0.926)$ & $(0.943)$ & $(0.944)$ \\
\hline \multirow{2}{*}{ Job Motivation } & Experimental & 48.60 & 4.42 & 101.30 & 8.04 & $<0.001$ & $<0.001$ & $<0.001$ \\
\hline & Control & 52.95 & 5.61 & 52.75 & 9.07 & $(0.801)$ & $(0.912)$ & $(0.913)$ \\
\hline $\begin{array}{l}\text { Positive } \\
\text { Organizational }\end{array}$ & Experimental & 37.95 & 4.94 & 85.05 & 5.24 & $<0.001$ & $<0.001$ & $<0.001$ \\
\hline Behavior & Control & 39.60 & 3.66 & 38.50 & 3.47 & $(0.906)$ & $(0.950)$ & $(0.954)$ \\
\hline
\end{tabular}

the variable of positive organizational behavior, it was ( $p$ $=0.169)$ in pre-test, and it was $(p=0.07)$ in post-test, therefore the assumption of homogeneity of variance was not rejected. The assumption of homogeneity of variance covariance matrix was also confirmed by Box test in all three variables of job stress $(p=0.137)$, job motivation ( $p$ $=0.748)$ and positive organizational behavior $(p=0.223)$.

Based on the results of analysis of variance with repeated measures, the assumption of the equality of mean scores in two stages of pre-test and post-test in the variables of job stress $(F(1,38)-623.97, p<0.2 \eta, 001-$ $0.943)$; job motivation $(\mathrm{F}(1,38)-391.78, \mathrm{p}<0.2 \mathrm{\eta}, 001-$ $0.912)$; and positive organizational behavior $(\mathrm{F}(1,38)$ 717.32, $\mathrm{p}<0.2 \eta, 001-0.950)$ was rejected. The effect of experimental group on error level of $5 \%$ for the variables of job stress $(\mathrm{F}(1,38)-475.062, \mathrm{p}<0.2 \mathrm{n}, 001$ $0.926)$; job motivation $(\mathrm{F}(1,38)-153.20, \mathrm{p}<0.2 \mathrm{\eta}$, 001$0.801)$; and positive organizational behavior $(\mathrm{F}(1,38)-$ $366.193, \mathrm{p}<0.2 \eta, 001-0.906)$ was significant. Also, the interaction effect between the experimental group and the measurement time on variables of job stress $(\mathrm{F}(1,38)$ -646.54, $\mathrm{p}<0.2 \mathrm{\eta}, 001-0.944)$; job motivation ( $\mathrm{F}(1,38)$ $-623.97, \mathrm{p}<0.2 \mathrm{n}, 001-0.943)$; and positive organizational behavior $(\mathrm{F}(1,38)-623.97, \mathrm{p}<0.2 \eta, 001-0.943)$ at error level of $5 \%$ was meaningful.

The result of Bonferroni post hoc test on the interaction effect on job stress showed no significant difference in the control group in the two stages of pre-test and post-test ( $p$ $=0.753$ ) but in the training group, teachers' job stress had a significant decrease in post-test compared to pre-test ( $p$ $<0.001$ ). In addition, in inter-group comparison, it was found that in pre-test, there was no significant difference in teachers' job stress between the experimental and control groups $(\mathrm{p}=0.418)$, but after the intervention, the level of job stress was significantly lower in the experimental group, the job stress was significantly lower than the control group $(\mathrm{p}<0.001)$.

Considering the variable of job motivation, in the control group the mean scores of job motivation in two stages of pre-test and post-test were not significantly different $(p=0.916)$, but in the training group, the teachers' job motivation in post-test was significantly increased compared to pre-test $(\mathrm{p}<0.001)$. In addition, it was found that in pre-test, the job motivation of the teachers in the experimental group was significantly lower than the control group $(p=0.010)$, but after the intervention, the job motivation in the teachers of the experimental group was significantly higher than the control group $(\mathrm{p}<0.001)$.

Regarding the variable of positive organizational behavior it was shown that in the control group, the mean score of organizational behavior in the two stages of pretest and post-test was not significantly different (p- 0 . 350 ), but in the training group of teachers, the positive organizational behavior in post- test was significantly increased compared to the pre-test ( $p<0.001)$. Additionally, in inter-group comparison, it was found that in the pre-test phase, considering the organizational behavior, there was no significant difference between the control group and the experimental group ( $p-0.223$ ), but after the intervention, the organizational behavior in the experimental group was significantly higher than the control group.

\section{Discussion}

The findings of the study showed that Neuro-linguistic programming has a significant effect on the improvement of positive organizational behavior, reduction of job stress and extension of job motivation. It is very important that how employees interpret and analyze the intraorganizational events and the incidents in their own lives. There may be many positive and negative events in the lives of all individuals and all employees in the organization, but our perceptions of these events are more important. Neuro-linguistic programming helps employees to interpret daily events and events in a way that minimizes stress and tension and provides a better and positive view of life. This will improve their behaviors and make them show more positive and effective behavior in the organization, which is confirmed by the findings of the study.

In other words, after learning the components of Neuro-linguistic programming, they learn how to better communicate with others, control their own inputs, and evaluate events in a positive way so they can have a higher level of motivation and a lower level of stress and tension in the organization, in other words school. In other words, people trained in Neuro-linguistic programming in this study learned how to better communicate with others and 
with the environment and to have a better understanding of colleagues, individuals and students, and even events, and this helps the physical education teachers who are actively in contact with students in the realm of sport to have more control over their own minds and others' perceptions while teaching, and be able to manage their thoughts in a positive way, and not to allow stress and tensions and negative atmosphere to increase in the mind.

Job stress and Neuro-linguistic programming were correlated based on the findings of the study and after the end of the course of Neuro-linguistic programming, the level of job stress in teachers was significantly reduced. Job stress can greatly affect the performance of physical education teachers and create other psychological problems at home and outside the workplace, which is a good solution to manage this stress was Neuro-linguistic programming. In the presented course, the concepts of self-worth and self-love were presented to the teachers, which makes the teachers of physical education consider their job worthy despite all the problems and value their job and their social status as teachers, and manage their stress better in this way. Mental toughness and internal control can be largely effective in controlling the difficulties and the problems that are induced by one's psyche, and physical education teachers feel less stressed by the lessons presented. Job stress is a component whose control and amount depends on the spiritual and mental management in individuals, and Neuro-linguistic programming can help teachers to experience a lower level of job stress. In other words, physical education teachers whose inclusion criterion was being exposed to high levels of job stress had experienced less stress after the end of the course and experienced less stress indicating that they had been able to control the stress by using the training provided.

Positive organizational behavior was another variable examined in this study. The components of positive organizational behavior consisted of optimism, hope, resilience, and self-efficacy consistent with the headlines of the training course, and we observed an increase in this variable among the participants of the study. Selfmotivation and positive thinking and the concepts presented in the relevant sessions are highly consistent with the optimistic and hopeful statements in positive organizational behavior that result in a significant increase in this variable in the post-test period. Setting goal and increasing self-confidence, and effective relationship were the indicators emphasized in the course of the training, and self-efficacy and its elements are in line with these components. In other words, it can be said that Neuro-linguistic programming has a positive effect on creating positive organizational behaviors in physical education teachers and in this regard, physical education teachers can optimally and hopefully cope with internal and external problems in the workplace and enhance their effectiveness in the organization and non-work environments, and Neuro-linguistic programming is an effective factor in this regard.

Job motivation is also a factor which has been discussed and examined in the teachers, and many studies have highlighted the lack of motivation of teachers in various societies and majors. In this study, pre-test showed a low level of job motivation in teachers of physical education and on the other hand, it was found that job motivation had a positive and significant increase under the influence of Neuro-linguistic programming. Job motivation as the main driving force in the job of physical education teachers determines their performance and shows to what extent they perform their job with interest and motivation. The topics taught in Neuro-linguistic programming, including self-esteem, emphasized on the importance of the job of physical education teachers and emphasized on their motivation and interest in their work and taught them to value themselves and their jobs. Selfmotivation and hope, positive thinking, self-efficacy, and utilizing the intrinsic abilities were the concepts presented during this period, increasing the motivation of physical education teachers in their jobs was emphasized, and it was tried to train physical education teachers to be positive about themselves and their job and have more hope and motivation in their jobs and lives.

Based on the findings of Ray et al. [14] job stresses decreases the life quality, and tools like Neuro-linguistic programming are needed to manage these stresses properly in individuals in order not to cause mental and personality crises. In their research, Ahmadi et al. [7] and Kajbaf et al. [17] and Mikaili et al. [18] also found Neurolinguistic programming effective in reducing tension, stress and anxiety and mental disorders and improvement of adaptation of individuals which is in line with the findings of this research. In other words, people who can control their mental inputs and effectively adapt to other people and organizations, and this concept is in line with the concept of positive organizational behavior and, on the other hand, the effect of Neuro-linguistic programming on improving mental tranquility and reducing stress which can be considered to be associated with a decrease in job stress that results in increased job motivation in physical education teachers. Ashok \& Santakumar [19], Thompson et al. [20] and Grosu et al. [21] also emphasize on the importance of Neuro-linguistic programming and its effective role in improving learning, performance development, and enhancing the decision making skills in individuals which is consistent with the findings of this study. Lashkarian and Sayyadian [22] also believe that for better communicating with students, a teacher can improve university students' effectiveness, motivation, and skill through Neuro-linguistic programming, enhancing learning environments, and a positive interaction, and the present study also emphasizes on the importance of Neuro-linguistic programming in the process of education for teachers, students and university students. Florina et al. [23] also found that Neuro-linguistic programming is effective in reducing anxiety and improving information inputs, which is in line with the findings of this study. Yemm [24] believes that Neuro-linguistic programming can be effective for businesses and can be damaging when damaging cases are related to employees quitting the job 
due to their increased ability and feeling superior to the organization level which is inconsistent with findings of this study. But in physical education teachers, Neurolinguistic programming can be considered as an effective factor, as the educational area differs from the business environment especially in European countries, and physical education teachers through reducing tension and anxiety and job stress and developing self-efficacy and control skills, and consequently positive organizational behavior can develop skills related to Neuro-linguistic programming and experience higher job motivation, and their performance can be effectively increased. Teaching Neuro-linguistic programming along with emphasizing on skills like effective communication and improving selfconfidence can increase the interest of physical education teachers in their jobs and even their personal lives, and make them internally plan for reducing the focus on problems and mental reconstruction. Based on the topics presented in this course, physical education teachers have learned to set a different goal for their job and be able to cope with toughness and mental problems, and provide positive motivation to themselves, and these solutions improved their mental state and psychological readiness, and based on the findings of this research, it leads to the improvement of their motivation and relieve from job stress, and considering the training of communication skills and effectively understanding others, teachers' behavior with others, including students, colleagues and individuals outside the organization is promoted, and the development of positive behavior in teachers is enhanced.

\section{Conclusion}

Based on the findings of the study, it is suggested that although organizational and life problems and personal problems of teachers require macro planning and national support and determination, education managers can improve organizational behaviors by conducting useful Neuro-linguistic programming courses and reduce the stress of job stress in physical education teachers. In other words, it is recommended that NLP courses be held during the academic year for in-service physical education teachers and by setting a rating for these courses, so that teachers can benefit from these trainings and develop positive organizational behaviors. These courses are also effective in reducing job and psychological stress and relieving the mind of the teachers. It is recommended that courses be held for teachers. Based on the research findings, in this section, organization-supervised courses as well as courses outside the organization framework sponsored by the organization are recommended for physical education teachers. These courses, with the topics presented in this study, can increase the focus of physical education teachers on internal management and control of problems, and it is recommended that courses with these topics are held for the teachers throughout the year.

\section{Conflict of interest}

The authors declare that there is no conflict of interest.

\section{References}

1. Karimi A, Rezazadeh H, Mortazavi S. The Effect of Psychological Capital of the Organization's Employees on their Emotional and Psychological Well-being: The Role of Positive Excitement, Stress and Attraction. Research on Organizational Resource Management, 2013; 3(3):100-106.

2. Bandura A. An agentic perspective on positive psychology. In: Lopez SJ, ed. Positive psychology: Exploring the best in people. Westport, CT: Greenwood Publishing Company; 2009.

3. Bakker AB, Schaufeli WB. Positive organizational behavior: engaged employees in flourishing organizations. $J$ Organiz Behav ,2008;29:147-54. https://doi.org/10.1002/job.515

4. Nezafatshoar F. The Relationship between Job Stress and Job Performance with the Moderating Role of Emotional Intelligence in Nurses. Faculty of Literature and Humanities, Payame Noor University, Fars Province; 2011.

5. Nisi K, Arshadi N, Rahimi E. The causal relationship between psychological capital with positive emotion, psychological well-being, job performance and job engagement. Journal of Psychological Achievements, 2011; 4(1): 46-49.

6. Troesch LM, Eve Bauer C. Second career teachers: Job satisfaction, job stress, and the role of self-efficacy. Teaching and Teacher Education, 2017; 67: 389- 398. https://doi.org/10.1016/j.tate.2017.07.006

7. Ahmadi R, Ahadi H, Mazaheri M, Delavar A, Najarian B. The Effect of Teaching Nervous Programming on Students' Depression. New Findings in Psychology, 2011; 2(18):2329.
8. Pan X, Mao T, Zhang J, Wang J, Su P. Psychological capital mediates the association between nurses' practice environment and work engagement among Chinese male nurses. International Journal of Nursing Sciences, 2017;4:378-83. https://doi.org/10.1016/j.ijnss.2017.09.009

9. Woo CH, Park J Y. Specialty satisfaction, positive psychological capital, and nursing professional values in nursing students: A cross-sectional survey. Nurse Education Today, 2017; 57: 24-28. https://doi.org/10.1016/j.nedt.2017.06.010

10.Bacon, Stephen C. Neurolingustic programming and psychosomatic illness:a study of the effects of reframing on headache pain [Dissertation abstracts]. University of Montan; 2017; 44(7): 2233b.

11.Hossack A, Standige K. Using using and imagery scrapbook for neurolingustic programming in the after match of a clinical depression: a case history sorce. Gerontologist, 2013, 33: 265-268. https://doi.org/10.1093/geront/33.2.265

12.Stipanic M, Enner W, Scha P. Effect on neurolinguistic psychotherapy on psychological difficulties and perceived quality of life. Counseling and Psychotherapy Research, 2010; 10: 39-49. https://doi.org/10.1080/14733140903225240

13.Jancenelle VE, Javalgi R (Raj) G, Cavusgil E. The role of economic and normative signals in international prosocial crowdfunding: An illustration using market orientation and psychological capital. International Business Review, 2018;27:208-17. https://doi.org/10.1016/j. ibusrev.2017.07.002 
14.Ray TK, Kenigsberg TA, Pana-Cryan R. Employment arrangement, job stress, and health-related quality of life. Safety Science, 2017;100:46-56. https://doi.org/10.1016/j.ssci.2017.05.003

15. Woo CH, Park JY. Specialty satisfaction, positive psychological capital, and nursing professional values in nursing students: A cross-sectional survey. Nurse Education Today, 2017; 57: 24-28. https://doi.org/10.1016/j.nedt.2017.06.010

16. Younesian A, Kalalian Moghaddam H, Mohammadiyoun M. Designing an Aerobic Exercise Model in Water and Its Role in Depression Improvement. Journal of Knowledge and Health, 2016; 2(2):45-52.

17.Kajbaf M, Moghaddas M, Aghaei A. The Effectiveness of Strategic Training of Verbal Neural Planning on Marital Adaptation. Knowledge and Research in Applied Psychology, 2010; 12(4):56-62.

18.Mikaeli F, Hemati M, Farhadi M, Fayyipour, Hayedeh. The Effect of Neuro-Verbal Planning on Mental Health of Urmia Specialist Nurses. Urmia Journal of Nursing and Midwifery, 2015; 14 (5): 437-445.

19.Ashok S, Santakumar AN. NLP to promote TQM for effective implementation of ISO 9000. Managerial Auditing Journal, 2016; 7(15);34-40.

20.Thompson JE, Courtney L, Dickson D. The effect of neurolinguistic programming on organisational and individual performance: a case study. Jnl Euro Industrial Training, 2002;26:292-8. https://doi.org/10.1108/03090590210431265
21.Grosu EF, Preja CA, Iuliana BB. Neuro-linguistic Programming Based on the Concept of Modelling. Procedia - Social and Behavioral Sciences, 2014; 116: 3693 - 3699. https://doi.org/10.1016/j.sbspro.2014.01.825

22.Lashkarian A, Sayadian S. The Effect of Neuro Linguistic Programming (NLP) Techniques on Young Iranian EFL Learners' Motivation, Learning Improvement, and on Teacher's Success. Procedia - Social and Behavioral Sciences, 2015;199:510-6. https://doi.org/10.1016/j.sbspro.2015.07.540

23.Florina GE, Teodor GV, Cornelia PS, Marin D. Neurolinguistic Programming and the Relationship between Attention and Anxiety in Alpine Skiing Juniors. Procedia Social and Behavioral Sciences, 2015; 191: 1634 - 1638. https://doi.org/10.1016/j.sbspro.2015.04.577

24.Yemm G. Can NLP help or harm your business? Ind and Commercial Training, 2006;38:12-7. https://doi.org/10.1108/00197850610645990

25.Steinmetz JI, Kaplan RM, Miller GL. Stress management: an assessment questionnaire for evaluating interventions and comparing groups, J Occup Med, 1982;24(11):923-31.

26.Luthans F, Youssef CM, Avolio BJ. Psychological capital: Developing the human competitive edge. Oxford, UK: Oxford University Press; 2007.

27.Hackman JR, Oldham GR. Motivation through the design of work: Test of a theory. Organizational Behavior and Human Performance, 1976;16: 250-279.

\section{Information about the authors:}

Anjomshoa M.R.; http://orcid.org/0000-0002-7132-7751; anjom_reza@yahoo.com; Department of Physical Education And Sport Sciences, Mashhad Branch, Islamic Azad University; Mashhad, Iran.

Fahim Devin Y.; (Corresponding Author); http://orcid.org/0000-0001-7901-0129; fahim_devin@yahoo.com; Department of Physical Education And Sport Sciences, Mashhad Branch, Islamic Azad University; Mashhad, Iran.

Esmailzadeh M.R.; http://orcid.org/0000-0001-8691-429X; esmailzadehm50@yahoo.com; Department of Physical Education And Sport Sciences, Mashhad Branch, Islamic Azad University; Mashhad, Iran.

Keshtidar M.; http://orcid.org/0000-0002-1705-4940; keshtidar@um.ac.ir; Faculty of Sport Sciences, Ferdowsi University of Mashhad; Mashhad, Iran.

Cite this article as:

Anjomshoa MR, Fahim Devin Y, Esmailzadeh MR, Keshtidar M. Effects of neuro-linguistic programming course on job stress, positive organizational behavior and job motivation in physical education teachers. Pedagogy of physical culture and sports, 2020;24(3):111-117. https://doi.org/10.15561/26649837.2020.0302

This is an Open Access article distributed under the terms of the Creative Commons Attribution License, which permits unrestricted use, distribution, and reproduction in any medium, provided the original work is properly cited (http://creativecommons.org/licenses/by/4.0/deed.en).

Received: 20.11.2019

Accepted: 26.12.2019; Published: 05.01.2020 